\title{
A non-linear static approach for the prediction of earthquake-induced deformation of geotechnical systems
}

\author{
R. Laguardia ${ }^{1}$ (D) $\cdot$ D. Gallese ${ }^{1}$ (D) $\cdot$ R. Gigliotti ${ }^{1} \cdot$ L. Callisto $^{1}$ (D)
}

Received: 30 March 2020 / Accepted: 30 August 2020 / Published online: 21 September 2020

(c) The Author(s) 2020

\begin{abstract}
This paper illustrates an original and simple method to predict earthquake-induced deformations of geotechnical systems. The method is an extension of static non-linear analysis, and is conceived to predict the behaviour of geotechnical systems, like supported and unsupported excavations, that during the seismic motion accumulate displacements in a single direction. The seismic capacity of the system is described by its capacity curve, obtained either from a numerical push-over analysis or through a simplified procedure. The corresponding seismic demand is described by a combination of the elastic response spectrum, including basic information on the maximum amplitudes of the seismic motion, and a cyclic demand spectrum, that provides additional information about the equivalent number of cycles that contribute to the accumulation of displacements. In the paper, the method is described in detail and is validated through different procedures, namely: comparisons with experimental results obtained in the geotechnical centrifuge; comparison with results of advanced numerical analyses; extensive comparison, using a large database of seismic records, with the results of non-linear time-domain analyses. In its final part, the paper provides guidance for the practical use of the method for design.
\end{abstract}

Keywords Seismic design · Retaining structures · Earthquake-induced displacements · Cyclic demand $\cdot$ Ground motions

\section{Introduction}

When subjected to seismic actions, many geotechnical systems are characterised by a strongly asymmetric behaviour. Some examples are provided in Fig. 1: slopes, unsupported excavations, and different types of retaining structures are all subjected to gravity forces that tend to displace the soil and the structural members towards the weaker zone of the system (namely, to the left in Fig. 1). Because of this asymmetry, during a severe earthquake these systems tend to accumulate irreversible deformations, mostly deriving from the transient activation of their global resistance. It is for this reason that

R. Laguardia

raffaele.laguardia@uniroma1.it

1 Department of Structural and Geotechnical Engineering, Sapienza University of Rome, via Eudossiana 18, 00184 Rome, Italy 

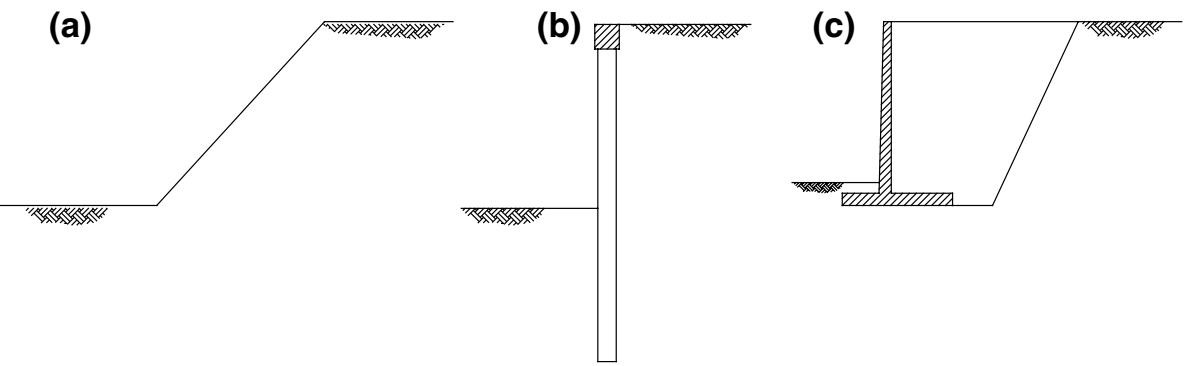

Fig. 1 Examples of displacing retaining systems: a unsupported excavation; b embedded retaining structure; c gravity retaining structure

the seismic performance of these geotechnical systems is often assessed on the basis of their final deformation (e.g. Richards and Elms 1979).

In the past, it has been proposed to compute the final displacements of these systems through a sliding-block analysis. This was originally suggested by Newmark (1965) for the seismic analysis of earth dams and was subsequently extended by many authors to the analysis of different geotechnical systems, including unsupported excavations (e.g. Franklin and Chang 1977), retaining structures (e.g. Richards and Elms 1979; Wong 1982; Whitman 1990) and foundations (e.g. Richards et al. 1993, Callisto and Rampello 2013). In addition, various correlations were developed between the global resistance of the system and different ground motion parameters (Ambraseys and Menu 1988; Rathje et al. 1998).

Basically, a sliding-block approach implies the assumption of a rigid-perfectly plastic behaviour and therefore neglects the dynamic response of the system under consideration. To some extent this limitation can be addressed using decoupled procedures, like those originally proposed by Seed and Martin (1966) and by Chopra (1966) and further developed, for instance, by Rathje and Bray (1999) and Baziar et al. (2012). In this decoupled approach the input signal used to integrate the equation of motion for a rigidperfectly plastic system is evaluated from a free-field ground response analysis that accounts for the deformability and non-linearity of the soil. In turn, the ground motion evaluated in the free-field is applied as an input motion to a rigid-perfectly plastic model of the geotechnical system to evaluate its displacements. It is evident that this procedure considers the deformability that the soil exhibits in the free-field, but cannot account for the deformations that accompany the progressive activation of the resistance of the system. In fact Callisto (2014), analysing the results of non-linear numerical analyses and examining the stress-paths of different soil elements located near a retaining wall, concluded that the pre-failure deformability shown by the soil in the transition from the static initial condition to the activation of a plastic mechanism has a significant influence of the computed displacements.

An additional problem with the sliding block analysis is that the displacements induced by the earthquake are computed with a time-domain integration, and therefore it is necessary to express the seismic action in terms of acceleration time-histories: while this is feasible for the non-linear analysis of important structures or infrastructural systems, often the geotechnical systems of the type shown in Fig. 1 are ordinary structures, for which the seismic action is taken directly from the construction codes and is expressed in terms of uniform-hazard elastic response spectra. As mentioned 
above, simplified methods relating displacements to ground motion parameters do exist, but each of them was developed for specific sets of accelerograms that can hardly be deemed representative of the various seismic hazard situations encountered in design.

To overcome the above limitations, the present paper illustrates an original method for the evaluation of the permanent displacements of the geotechnical systems of the type illustrated in Fig. 1, with a specific emphasis on the case of earth retaining structures. The method extends the common procedures for non-linear static analysis (Freeman et al. 1975; Fajfar 2000), originally developed for buildings and bridges, to the prediction of the cyclic accumulation of displacement relevant for the geotechnical systems at hand. In the method proposed herein, the seismic capacity of the system is represented by a non-linear capacity curve, as in a static non-linear analysis, while the seismic demand is represented by a combination of an elastic response spectrum and an additional response spectrum, that expresses the number of equivalent cycles of the seismic action as a function of the natural period of a single-degree-of-freedom system.

The illustration of the method is preceded by a description of the typical dynamic response of the systems under consideration, that is addressed in the next section.

\section{Dynamic response of geotecnhical systems}

\subsection{Capacity curve}

An effective way to evaluate the seismic capacity of a geotechnical system, proposed by Callisto (2019), consists in applying equivalent inertial forces to a non-linear numerical model of the system. The inertial forces are taken to be proportional to a seismic horizontal coefficient $k_{\mathrm{H}}$, that represents the ratio of the horizontal body forces to the unit weight of the soil or, equivalently, the ratio of the horizontal acceleration to the gravity acceleration. Figure 2 shows for an embedded retaining wall the relationship obtained by Callisto (2019) between the seismic coefficient $k_{\mathrm{H}}$ and the corresponding horizontal displacement of the top of the wall $u_{\mathrm{R}}$, expressed as a fraction of the excavation height

(a)

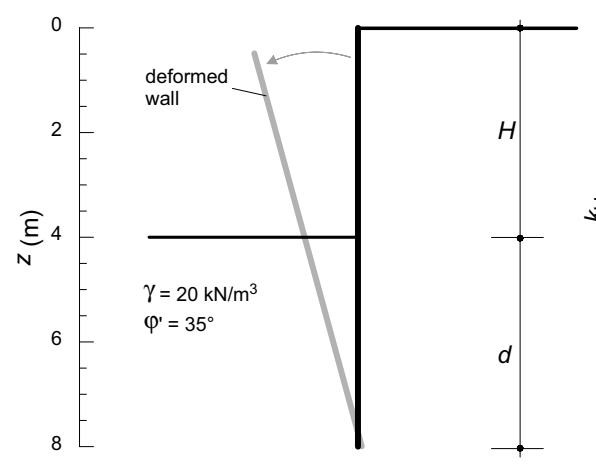

(b)

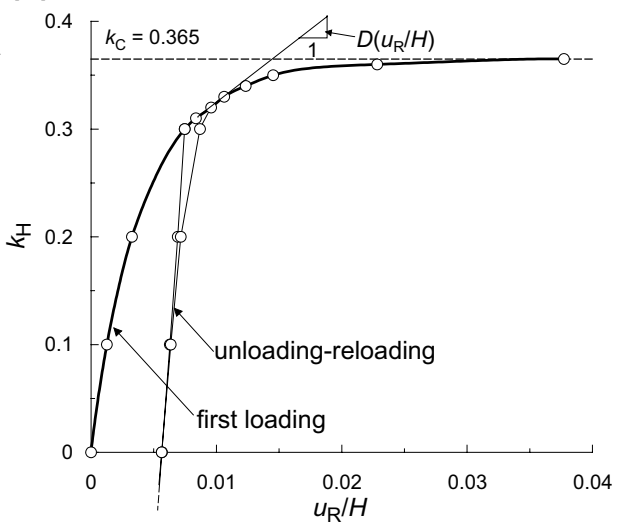

Fig. 2 a Example embedded retaining wall $\left(\gamma\right.$ is the unit weight of soil and $\varphi^{\prime}$ is the angle of shearing resistance); b non-dimensional capacity curve (Callisto 2019) 
$H$. The numerical analysis used to obtain this capacity curve was carried out with the finite difference method (code FLAC), regarding the soil as a non-linear elastic-perfectly plastic material, with a Mohr-Coulomb plasticity criterion and a non-associated flow rule with zero dilatancy. The main properties of the soil and the structural member are reported in Table 1. Further details of this type of analysis are given by Callisto (2014).

The plot in Fig. 2 can be regarded as a non-dimensional capacity curve for the retaining structure: it shows that the system deforms progressively, attaining its capacity for a horizontal displacement of the order of $1.5-2 \%$ of the excavation height. It can be demonstrated that, if the retaining structure is designed to remain in the elastic range throughout the loading process, most of the displacements exhibited by the wall derive in fact from the deformation of the soil, rather than from the deformation of the wall (Callisto and Soccodato 2010). Figure 2 also shows that on unloading-reloading the response is much stiffer, with a minor hysteresis loop, as a consequence of the strongly asymmetric behaviour of the system.

The ultimate capacity of the system can be expressed by the critical value $k_{\mathrm{C}}$ of the seismic coefficient, as indicated in Fig. 2. Callisto (2014) showed that $k_{\mathrm{C}}$ can be evaluated with a good accuracy not only from the results of a numerical analysis, but also using equilibrium equations together with solutions for the soil resistance derived from the theorems of limit analysis. Moreover, Callisto (2019) proposed to approximate the capacity curve using the following hyperbolic function:

$$
k_{\mathrm{H}}=\frac{s k_{C}}{s \alpha+s_{C}(1-\alpha)}
$$

where $s=u_{\mathrm{R}} / H$ is the normalised wall displacement, the coefficient $\alpha<1$ is the ratio of $k_{\mathrm{C}}$ to the asymptote of the hyperbolic function, and $s_{\mathrm{C}}$ is the normalised displacement needed to activate the collapse mechanism (Fig. 3). Callisto (2019) indicated that $\alpha$ and $s_{\mathrm{C}}$ can be related directly to the type of retaining structure under consideration: for instance, the capacity curve for embedded retaining structures may be characterised by values of $s_{\mathrm{C}}$ in the range of 0.015 to 0.020 , while for the same type of structure $\alpha$ varies from 0.8 to 0.9 .

The initial non-dimensional stiffness $D_{0}$ of the capacity curve can be evaluated differentiating Eq. (1), obtaining the expression:

$$
D_{0}=\frac{k_{C}}{s_{C}(1-\alpha)}
$$

showing that the normalised displacement $s_{\mathrm{C}}$ is related to the initial stiffness of the system. The unloading-reloading stiffness can be taken as $\beta D_{0}$, as shown in Fig. 3, where $\beta$ may vary from 1 to 2.5 (Callisto 2019).

Table 1 Soil and structural properties for the example case of Fig. 2

\begin{tabular}{llllll}
\hline$\gamma\left(\mathrm{kN} / \mathrm{m}^{3}\right)$ & $c$ & $\varphi^{\prime}\left(^{\circ}\right)$ & $\delta\left(^{\circ}\right)$ & $G_{0}(\mathrm{kPa})$ & $E I(\mathrm{kN} \mathrm{m} / \mathrm{m})$ \\
\hline 20 & 0 & 35 & 20 & $\begin{array}{l}10^{5} p^{0.5} \\
(p \text { in kPa })\end{array}$ & $2.7 \times 10^{5}$ \\
\hline
\end{tabular}

$\gamma$, unit weight; $c$, cohesion; $\varphi^{\prime}$, angle of shearing resistance; $\delta$, soilwall angle of friction; $G_{0}$, small-strain shear modulus; $p$, mean effective stress in the soil; $E I$, bending stiffness of the retaining structure 
Fig. 3 Hyperbolic approximation of a capacity curve, with an indication of the model parameters and of the specific work dissipated over one half-cycle

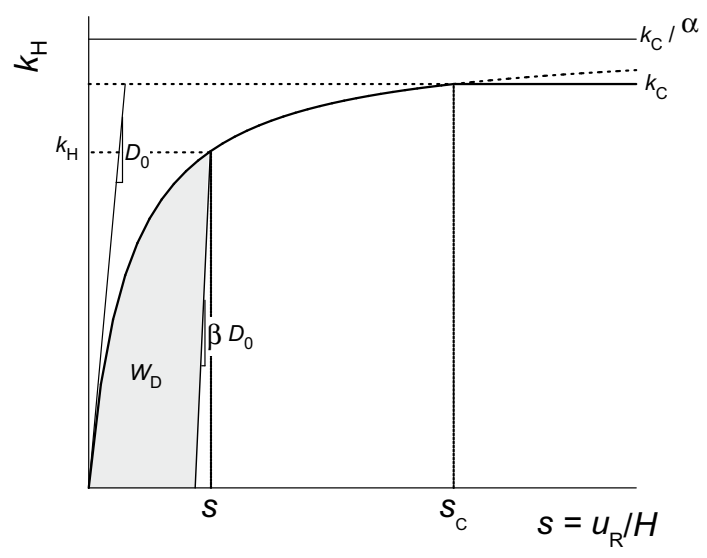

\subsection{Non-linear SDOF model}

Callisto (2019) demonstrated that the capacity curve defined above can be used to evaluate the seismic displacements of a retaining structure, performing a time-domain dynamic analysis of an equivalent non-linear system with a single degree of freedom (SDOF). Since the capacity curve is expressed in a non-dimensional form, it includes implicitly the dependence on $k_{\mathrm{H}}$ of the soil mass interacting with the structure (e.g. Richards and Elms, 1992). The equation of motion for the equivalent non-linear system can be expressed in a non-dimensional form as follows (Callisto 2019):

$$
\frac{\ddot{u}_{\mathrm{R}}(t)}{g}+2 \xi_{\text {ur }} \sqrt{\frac{D}{g H}} \dot{u}_{\mathrm{R}}(t)+\frac{D}{H} u_{\mathrm{R}}(t)=-\frac{a_{\mathrm{B}}(t)}{g}
$$

In Eq. (3), $H$ is the excavation height, $g$ is the gravity acceleration, $a_{\mathrm{B}}(t)$ is the seismic input, $u_{\mathrm{R}}(t)$ is the displacement of the top of retaining structure (or of the equivalent $\mathrm{SDOF}), \xi_{\mathrm{ur}}$ is an equivalent viscous damping ratio, and $D$ is the non-dimensional stiffness of the equivalent SDOF. The dot over the symbols denotes temporal derivation.

The stiffness parameter $D$ is defined as the local tangent to the capacity curve of the retaining system, evaluated either on the first loading branch of the curve, or along an unloading-reloading cycle. In the model, the viscous term of Eq. (3) is activated only along the unloading-reloading portions of the capacity curve, with a damping ratio $\xi_{\text {ur }}$ resulting from the interpretation of the typical unloading-reloading hysteresis loop, that for embedded retaining systems is of the order of $1 \%$. The seismic input $a_{\mathrm{B}}(t)$ is obtained from a free-field one-dimensional site response analysis, following the procedure proposed by Seed and Martin (1966). Figure 4 shows a typical response of the non linear SDOF subjected to a base motion (Callisto 2019). Specifically, Fig. 4a shows the time-histories of the input acceleration $a_{\mathrm{B}}(t)$ and of the corresponding acceleration response of the non-linear SDOF; Fig. $4 \mathrm{~b}$ shows the temporal evolution of the displacements of the system; and Fig. 4c depicts the progressive engagement of the capacity curve during the dynamic motion of the system. From the inspection of Fig. 4 the following comments can be made:

- The response of the non-linear SDOF is quite similar to that obtained from full non-linear dynamic analyses of the soil-structure domain (this is discussed in detail by Callisto 


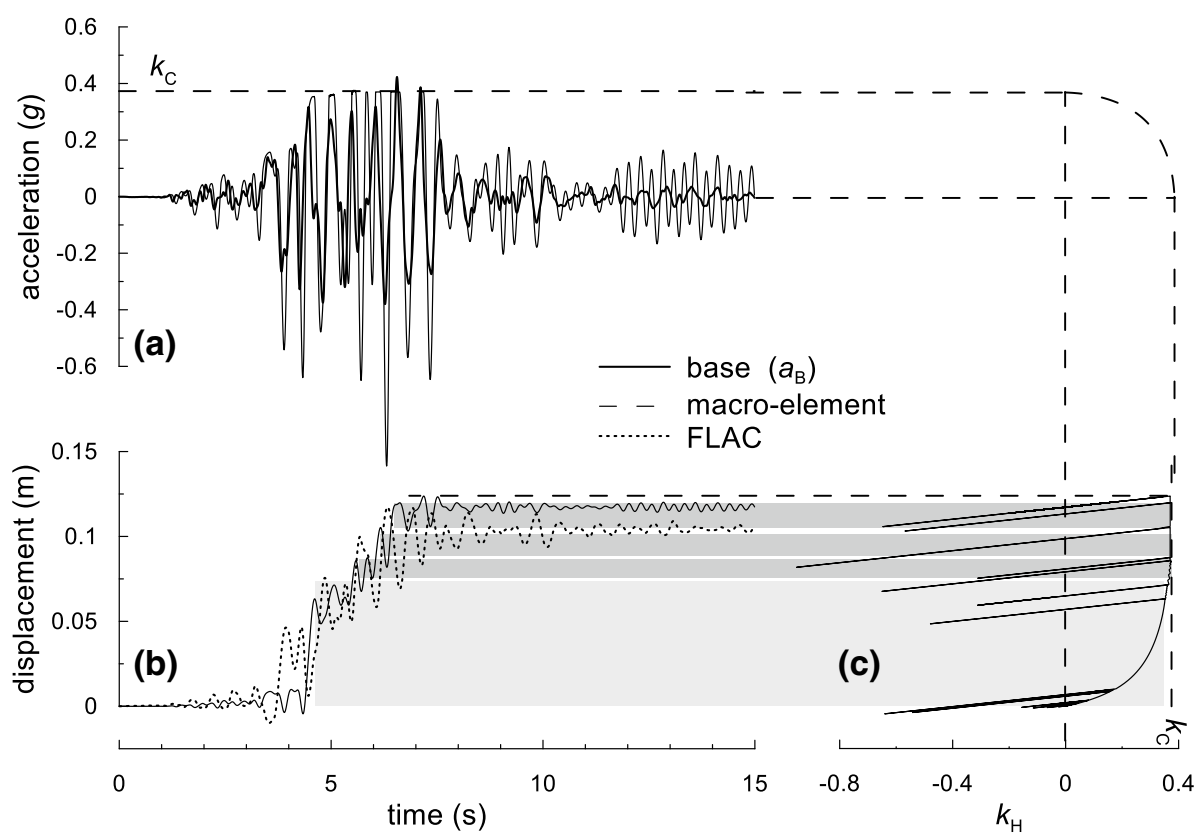

Fig. 4 Results obtained with the non-linear SDOF model: a input and output acceleration time histories; $\mathbf{b}$ time-history of the computed displacements; $\mathbf{c}$ progressive engagement of the capacity curve

2019). Therefore, in the following, the response of the non-linear SDOF will be taken as a reference for the development of the simplified approach.

- The deformability of the system has an effect on its dynamic response: this is visible, for instance, in the time interval of 4.5 to $6 \mathrm{~s}$, when the system reaches the critical acceleration while the input acceleration does not.

- The time history of the displacement can be conventionally sub-divided into two parts. In a first stage the system is brought to the activation of its capacity: for the example of Fig. 4, this happens roughly between 4.5 and $5.2 \mathrm{~s}$, as shown by the light shaded area. After this first activation, further displacements accumulate (as indicated by the dark shaded) that are triggered by the dynamic response along unloading-reloading cycles. These additional displacements occur only if the seismic motion is strong enough to activate the full capacity of the system.

The above observations provide the fundamental ingredients for the development of a simplified procedure, that permits an approximate evaluation of the final displacement of the system on the basis of the capacity curve and of the elastic response spectrum of the seismic action: this is explained in the next section.

\section{Layout of the simplified method}

The simplified method proposed in this paper is an extension of the common static nonlinear analysis used in earthquake engineering. 
Several methods may be used to perform a non-linear static analysis. The present work is based on the Capacity Spectrum Method (CSM), proposed and developed by Freeman et al. (1975), Freeman $(1998,2004)$ and adopted by many seismic codes and guidelines (e.g. ATC 1996; FEMA 2005, Circolare n.7 2019). According with the typical behaviour shown in Fig. 4 , the final displacement $u_{\text {fin }}$ is calculated as the sum of a first-activation displacement $u_{\mathrm{I}}$ and of a further component $u_{\mathrm{II}}$ deriving from the cyclic response of the system. In this second phase the system is considered as a SDOF with the stiffness of the unloading-reloading branch of the capacity curve.

The displacement $u_{\mathrm{I}}$ is obtained by superposing the capacity curve of the system and the Acceleration-Displacement (AD) elastic response spectrum of the seismic input. As in the original CSM, the equivalent damping ratio $\xi$ of the $\mathrm{AD}$ spectrum is found by iteration, i.e., by evaluating the damping ratio at the intersection of the capacity curve with the AD spectrum and re-plotting the spectrum accordingly. The damping ratio is evaluated from the equation:

$$
\xi=\frac{W_{\mathrm{D}}}{4 \pi W_{\mathrm{E}}}=\frac{W_{\mathrm{D}}}{2 \pi s k_{\mathrm{H}}}
$$

where $W_{\mathrm{E}}=0.5 s k_{\mathrm{H}}$ is the recoverable specific energy and $W_{\mathrm{D}}$ is the specific dissipated work, that because of the asymmetric behaviour of the systems at hand is taken equal to the shaded area in Fig. 3. The evolution of the damping ratio obtained with Eq. (4) is plotted in Fig. 5 as a function of the ratio $s / s_{\mathrm{C}}$. In this representation $\xi$ depends only on the unloading-reloading multiplier $\beta$. It can be seen that, as the normalized displacement $s$ approaches the value $s_{\mathrm{C}}$ corresponding to the system capacity, the damping ratio becomes of the order of $10 \%$.

The capacity curve and the AD spectrum plotted for this damping ratio intersect at a normalized displacement $s_{\text {int }}$, that can be either larger or smaller than $s_{\mathrm{C}}$, meaning that on the first activation the capacity of the system is or is not reached. These two cases are shown in Fig. 6a, b. Looking at the response of several non-linear SDOF models subjected to a variety of base motions, it was seen that on the first activation of the system capacity the typical displacement is not much larger than $s_{\mathrm{C}}$. Therefore, denoting the

Fig. 5 Damping ratio for different $\beta$ values as a function of normalized displacement of the system

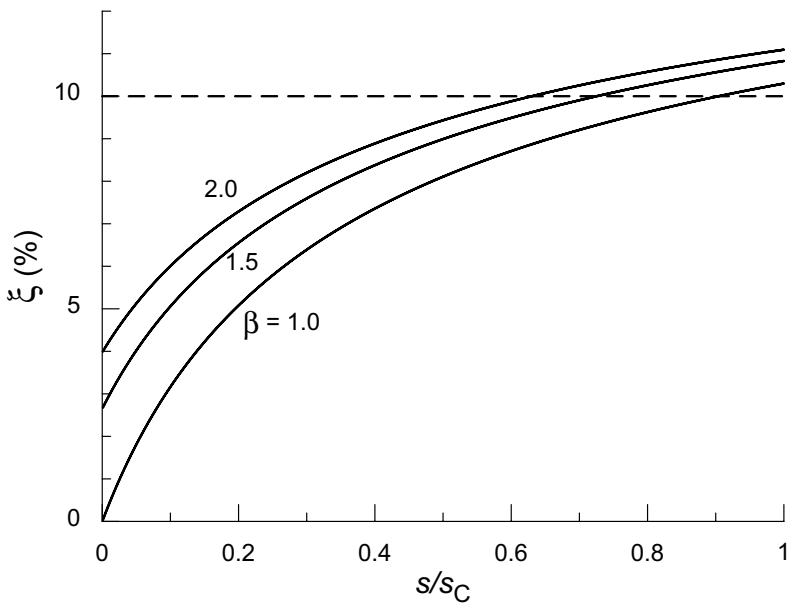


(a)

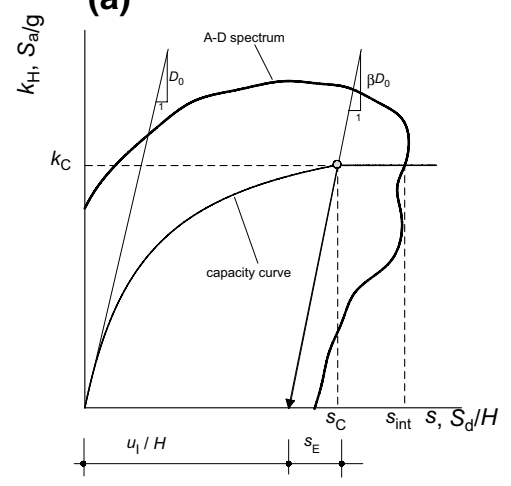

(c)

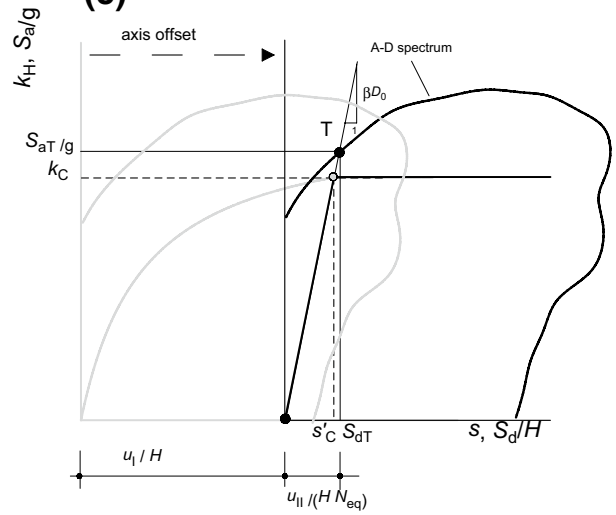

(b)

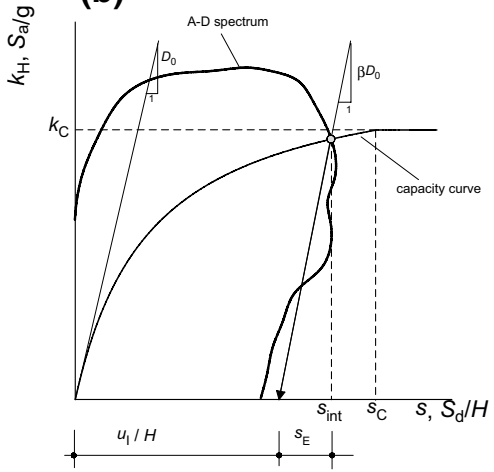

(d)

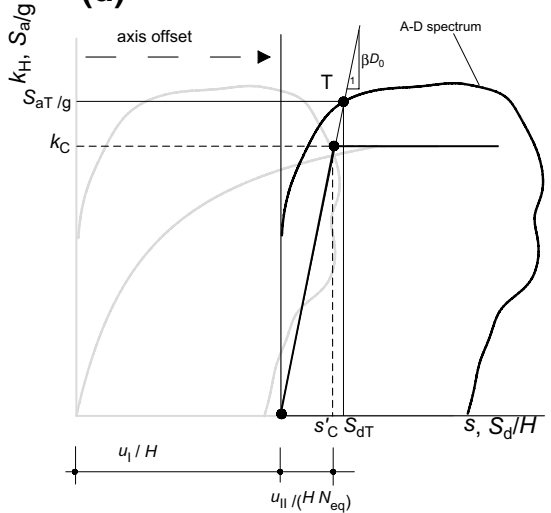

Fig. 6 Layout of the simplified method: calculation of first activation permanent displacement $u_{\mathrm{I}}$, for a $s_{\text {int }}>s_{\mathrm{c}}$, and $\mathbf{b} s_{\text {int }} \leq s_{\mathrm{c}}$; calculation of the threshold ratio $R_{\mathrm{T}}=g k_{\mathrm{C}} / S_{\mathrm{aT}}$ and the additional permanent $\mathrm{u}_{\mathrm{II}}$ if $\mathbf{c}$ $s_{\text {int }}>s_{\mathrm{c}}$ and $\mathbf{d} s_{\text {int }} \leq s_{\mathrm{c}}$

normalized elastic (recoverable) displacement as $s_{\mathrm{E}}$, the permanent displacement on first activation $u_{\mathrm{I}}$ is evaluated as:

$$
u_{\mathrm{I}}=H\left[\min \left(s_{\text {int }}, s_{\mathrm{C}}\right)-s_{\mathrm{E}}\right]
$$

Figure 4 shows that after the first activation the system can be taken to behave as a linearly elastic-perfectly plastic system, as shown in Fig. 6c, d, that can be analysed using the equal displacement rule (Veletsos and Newmark, 1960). Under this assumption, the additional displacements accumulated in this second phase are taken to depend on a threshold ratio:

$$
R_{\mathrm{T}}=\frac{g k_{\mathrm{C}}}{S_{\mathrm{aT}}}=\frac{s_{\mathrm{C}}^{\prime}}{S_{\mathrm{dT}}}
$$

where $s_{C}=k_{C} / \beta D_{0}$ is the normalized displacement needed to reach the capacity of the system along the unloading-reloading curve, while $S_{\mathrm{aT}}$ and $S_{\mathrm{dT}}$ are respectively the spectral 
acceleration and displacement corresponding to the elastic response of the system as shown in Fig. 6c, d.

The threshold ratio of Eq. (6) is the ratio of the critical acceleration to the spectral acceleration evaluated at the intersection of the elastic reloading branch of the capacity curve with the AD spectrum; or, equivalently, it is the ratio of the corresponding normalized displacements as shown in Fig. 6. If $R_{\mathrm{T}}$ is larger or equal to one, then no additional displacements occur, because it is assumed that after the first activation additional permanent displacements can happen only if the seismic action is able to activate the capacity of the system. If $R_{\mathrm{T}}$ is smaller than one, then the additional displacement $u_{\mathrm{II}}$ is evaluated as follows:

$$
u_{\mathrm{II}}=N_{\mathrm{eq}} S_{\mathrm{dT}}
$$

where $N_{\text {eq }}$ is the equivalent number of cycles of the seismic action that activate the capacity of the system. As such, $N_{\text {eq }}$ should be defined as a decreasing function of $R_{\mathrm{T}}$, as discussed in the next section. This additional displacement may occur even if $s_{\text {int }}<s_{C}$ (Fig. 6b), because on unloading the systems at hand show irreversible deformations and cyclic cumulated displacements even for $k_{\mathrm{H}}<k_{\mathrm{C}}$ (see Fig. 4c).

\section{Definition of equivalent cycles}

The definition of the equivalent number of cycles used in this work derives from the one proposed by Malhotra (2002): the number of equal-amplitude cycles that induces in a linearly elastic SDOF system the same damage of a given ground motion characterized by several half-cycles of different amplitude. The expression proposed by Malhotra (2002) for the number $N_{\text {cy }}$ of these equivalent cycles can be written as:

$$
N_{\text {cy }}=\frac{1}{2} \sum_{i=1}^{2 m}\left(\frac{u_{i}}{S_{\mathrm{d}}}\right)^{c}
$$

where $m$ is the number of half-cycles of absolute amplitude $u_{\mathrm{i}}$ undergone by the system subjected to the ground motion, and $S_{\mathrm{d}}$ is the maximum displacement of the system, that is, its spectral displacement. Since this expression derives from the Manson (1954) and Coffin (1954) fatigue model, Malhotra (2002) suggested that $c$ can be taken equal to 2, as commonly assumed for the fatigue models of steel specimens. The 1/2 factor in Eq. (8) is needed to transform the number of half-cycles considered in the summation assess into the corresponding number of equivalent full cycles.

In the simplified method proposed in this paper, it was seen that the displacement is accumulated by the system in two phases: an activation phase, that engages the first loading branch of the capacity curve, and an accumulation phase in which additional permanent displacements are triggered by the cyclic dynamic response along the unloading-loading branch of the capacity curve. In this second phase, the system is regarded as a linear SDOF system, similarly to the basic assumption of Eq. (8). Therefore, the number of equivalent cycles $N_{\text {eq }}$ was calculated with a relationship that retains the structure of the above equation, with the following modifications:

- there are no fatigue effects, so that successive displacements contribute equally to the seismic performance (i.e. $c=1$ ); 
- the summation is extended to the total number of peaks $n$, detected in the response of the equivalent SDOF to the input base motion;

- each cycle produces an additional displacement only if the ratio of its amplitude to the spectral displacement is larger than the threshold ratio $R_{\mathrm{T}}$;

- the $1 / 2$ factor is used to consider that the displacements are cumulated only on one-side: therefore, in the average only half of the counted cycles contribute to the deformation of the system.

Under these additional hypotheses, the expression for the equivalent number of cycles becomes:

$$
N_{\mathrm{eq}}=\frac{1}{2} \sum_{i=1}^{n}\left[\frac{u_{i}}{S_{\mathrm{dT}}} \times h s f\left(\frac{u_{i}}{S_{\mathrm{dT}}}-R_{T}\right)\right]
$$

where $h s f(x)$ is the Heaviside function (if $x>0 h s f(x)=1$; if $x \leq 0 h s f(x)=0$ ) and $S_{\mathrm{dT}}$ is the spectral displacement corresponding to the elastic response of the system, with a normalized stiffness equal to $\beta D_{0}$ (see Fig. 6c, d). As an example, Fig. 7 shows the displacement response of a linear SDOF system with a natural period $T_{0}=1 \mathrm{~s}$ subjected to the same ground motion record of Fig. $4 \mathrm{a}$. By considering a threshold ratio $R_{\mathrm{T}}=0.6$, it can be seen that only three peaks exceed the threshold displacement $u_{\mathrm{T}}=R_{\mathrm{T}} S_{\mathrm{dT}}$. In this case, Eq. (9) provides $N_{\text {eq }}=1.36$.

For application to the problem at hand, the natural period of the system can be found from the normalized stiffness in unloading-reloading. Observing that:

$$
\frac{S_{\mathrm{aT}}}{S_{\mathrm{dT}}}=\frac{4 \pi^{2}}{T_{0}^{2}}=\beta D_{0} \frac{g}{H}
$$

one obtains:

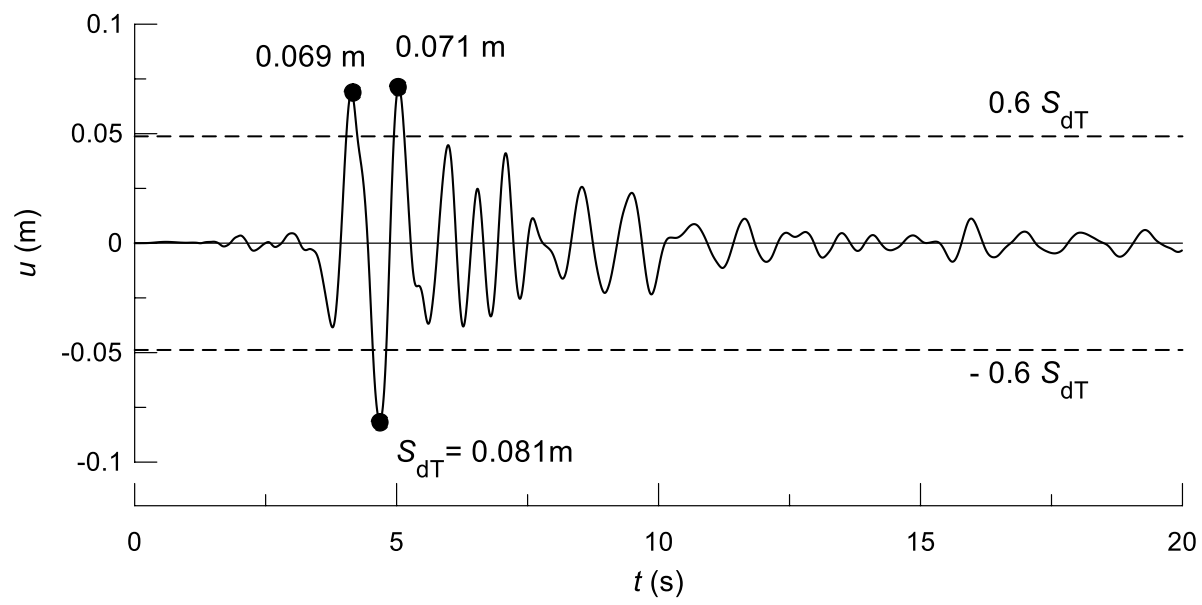

Fig. 7 Displacement history of a SDOF with $T=1 \mathrm{~s}$ subjected to the motion record of Fig. 4, with indication of the peak displacement with amplitude larger than $R_{\mathrm{T}} S_{\mathrm{dT}}$ 


$$
T_{0}=2 \pi \sqrt{\frac{H}{\beta D_{0} g}}
$$

that using Eq. (2) becomes:

$$
T_{0}=2 \pi \sqrt{\frac{s_{\mathrm{C}} H}{\beta k_{\mathrm{C}} g}(1-\alpha)}
$$

Equation (12) allows the calculation of the natural period of the system in unloading-reloading using the same set of parameters that characterise its capacity curve.

Figure 8 shows, for the same ground motion used in Figs. 4 and 7, a set of curves relating $N_{\text {eq }}$ to the SDOF natural period $T_{0}$ for several values of the threshold ratio $R_{\mathrm{T}}$. These curves, termed "cyclic demand spectra", were obtained for a damping ratio $\xi=10 \%$, as this is the value more commonly encountered in the application of the procedure (see Fig. 3). The shape of these curves is similar, but of course when the threshold ratio increases the equivalent number of cycles decreases.

As a further step, cyclic demand spectra of the type depicted in Fig. 8 were evaluated for a set of seismic records that could be deemed representative of the potential seismic actions of a given territory. Specifically, Eq. (9) was applied to the entire SIMBAD ground motion database Smerzini et al. (2014). This is a collection of 467 ground motion records, characterized by epicentral distances lower than $35 \mathrm{~km}$ and moment magnitudes ranging from 5 to 7.5, that was selected to represent effectively the seismic hazard of the entire Italian territory. Only a single horizontal component for each record was considered, given that the cyclic demand spectra obtained from two components of the same record were very similar.

As an example of the computation, Fig. 9 shows the envelopes of the cyclic demand spectra obtained for all the records of the database using two different threshold ratios $R_{\mathrm{T}}$, equal to 0.3 and 0.6. The plots of Fig. 9 also show for each period the average number of cycles and its scatter expressed as the values of $N_{\text {eq }}$ corresponding to plus or minus one standard deviation. The results are characterized by a coefficient of variation $(\mathrm{CoV})$ smaller

Fig. 8 Cyclic demand spectra obtained for the ground motion record of Fig. 4 by considering a damping ratio $\xi=10 \%$ and several threshold ratios $\mathrm{R}_{\mathrm{T}}$

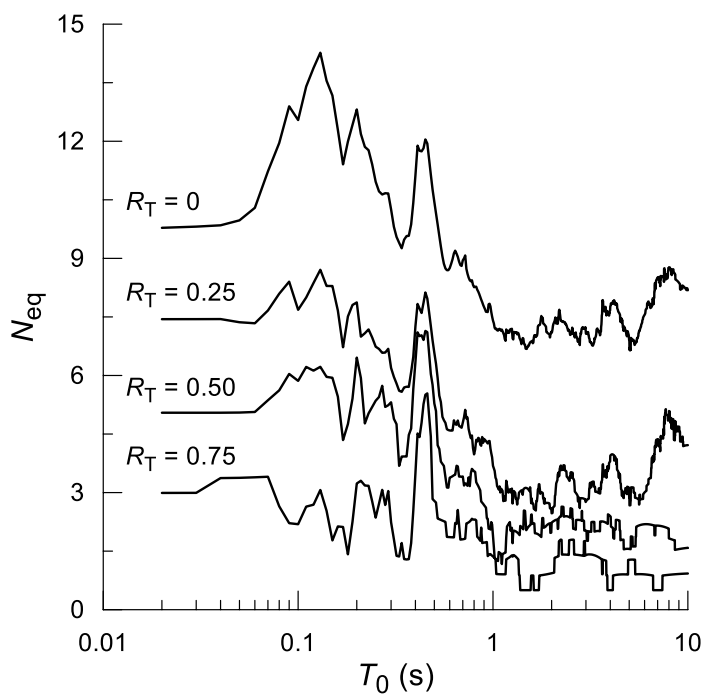



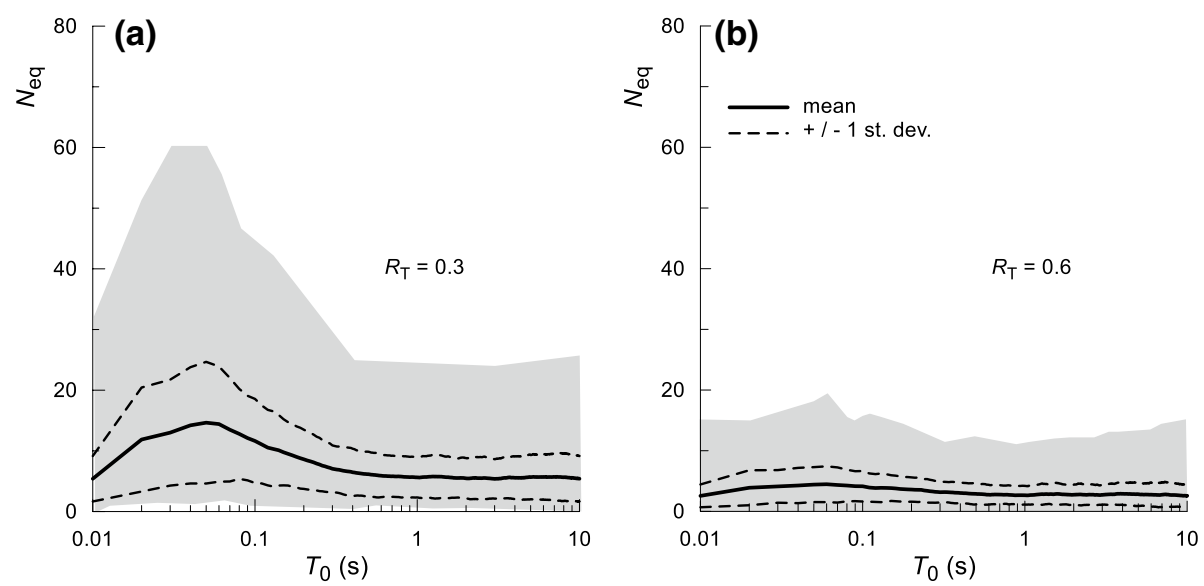

Fig. 9 Cyclic demand spectra obtained from the SIMBAD database, for a a threshold ratio $R_{\mathrm{T}}=0.3$, and $\mathbf{b}$ a threshold ratio $R_{\mathrm{T}}=0.6$

than 0.5 for $R_{\mathrm{T}}=0$ that increases for $R_{\mathrm{T}}>0$ up to values of about 0.8 . The number of equivalent cycles was seen to be only loosely correlated with the magnitude and distance of the seismic sources.

Figure 10 collects the average cyclic demand spectra obtained for threshold ratios $R_{\mathrm{T}}$ varying from 0 to 0.9 . These were all evaluated assuming a damping ratio $\xi=10 \%$, because by definition the accumulation of the displacements occurs as a result of the activation of the capacity of the system (see Fig. 5). For low threshold ratios $\left(R_{\mathrm{T}}<0.6\right)$ the average equivalent number of cycles $N_{\text {eq }}$ shows an appreciable variation with the vibration period

Fig. 10 Average cyclic demand spectra obtained from SIMBAD database for several threshold ratios ranging from 0 to 0.9

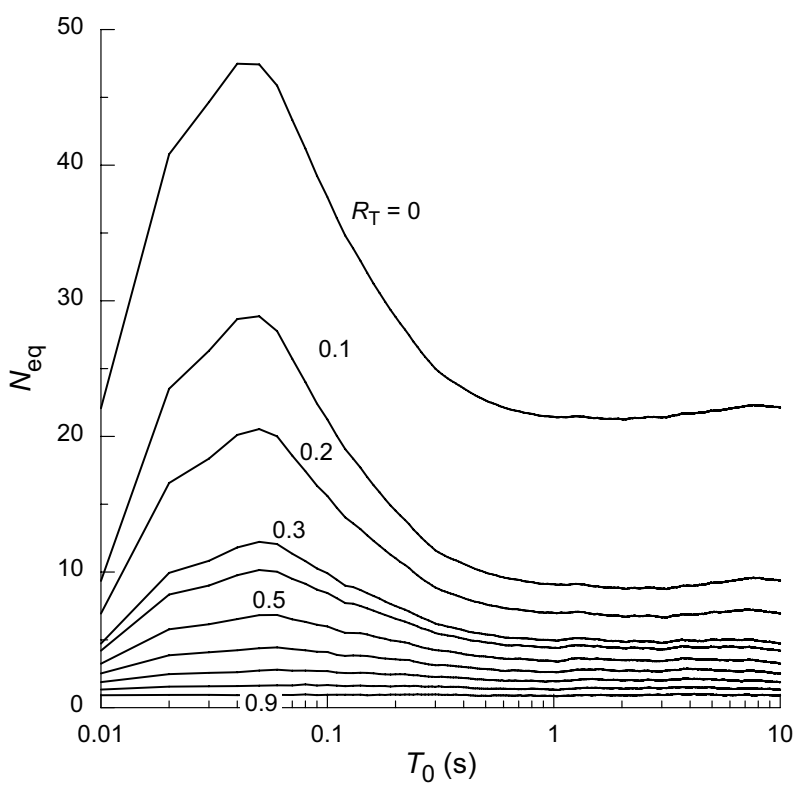


$T_{0}$ : it reaches a maximum at periods of about $0.06 \mathrm{~s}$, while at larger periods it decreases about linearly with the logarithm of $T_{0}$, remaining approximately constant for periods higher than $0.5 \mathrm{~s}$. Conversely, for threshold ratios larger than 0.5 the average equivalent number of cycles shows only a limited dependence on the period.

The average cyclic demand spectra of Fig. 10 were used to implement and to validate the simplified procedure outlined in the previous sections. The results of this validation are presented in the next section.

\section{Summary and validation of the procedure}

The procedure proposed in this paper is aimed at evaluating the seismic performance of geotechnical systems that during an earthquake may accumulate displacements because of the asymmetry of their response. In the simplified method presented herein the seismic demand is represented by the combination of an elastic response spectrum and a set of region-specific average cyclic demand spectra (one for each $R_{\mathrm{T}}$ value) as the one shown in Fig. 10. The seismic capacity is described by the capacity curve of the system, including the slope of the unloading-reloading branch. This may be evaluated either with a numerical push-over analysis of the system, or in an approximate way using readily available limit equilibrium methods (Callisto 2019). Once demand and capacity have been obtained, the seismic displacements can be calculated through the following steps:

1. Superimpose the capacity curve onto the elastic response spectrum in the accelerationdisplacement plane; this may require some iteration to find the appropriate value of the damping ratio; experience shows that a damping ratio of $10 \%$ is usually a good initial guess for cases of practical relevance.

2. Find the first-activation displacement $u_{\mathrm{I}}$ through Eq. (5).

3. Evaluate the threshold ratio $R_{\mathrm{T}}=g k_{\mathrm{C}} / S_{\mathrm{aT}}$ as shown in Fig. 6c, d.

4. If $R_{\mathrm{T}} \geq 1$ then there is no accumulation of deformations, and the permanent displacement $u_{\text {perm }}$ is equal to $u_{\mathrm{I}}$.

5. If $R_{\mathrm{T}}<1$ :

5.a. evaluate the unloading-reloading natural period $T_{0}$ from Eq. (12);

5.b. enter in the set of average cyclic demand spectra of Fig. 10 with $R_{\mathrm{T}}$ and $T_{0}$, and find $N_{\text {eq }}$;

5.c. $\quad$ compute the accumulated displacement $u_{\mathrm{II}}$ from Eq. (7);

5.d. compute the permanent displacement as the sum of $u_{\mathrm{I}}$ and $u_{\mathrm{II}}$.

This procedure was subjected to several validation steps. A first validation was carried out taking as a reference the results of a centrifuge experiment carried out by Conti et al. (2012), relative to embedded cantilevered walls that retained an excavation in a dry, coarsegrained soil. This centrifuge model was subjected to a base excitation consisting of a series of five quasi-harmonic signals, as shown in Fig. 11a. For this case, a capacity curve was evaluated with a FLAC 2D push-over analysis, using the information on the soil properties reported by Conti et al. (2012). This capacity curve is shown in Fig. 13: it is characterised by a critical coefficient $k_{\mathrm{C}}$ equal to 0.266 and by a corresponding normalised displacement $s_{\mathrm{C}}$ equal to 0.01 . To reproduce the observed behaviour, the input spectra of the five signals were obtained multiplying the actions applied at the base, having a dominant frequency of about $0.8 \mathrm{~Hz}$, by an amplification factor of $1.2 \mathrm{read}$ on the amplification curve derived 
Fig. 11 Interpretation of the centrifuge experiment by Conti et al. (2012) using the nonlinear SDOF and the simplified approach

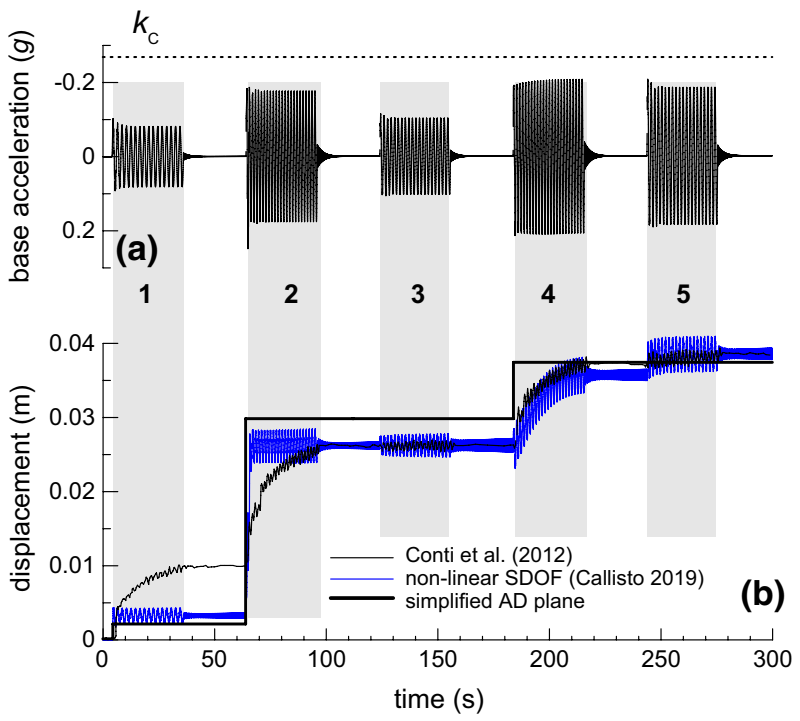

experimentally from the centrifuge experiment (Conti and Viggiani 2012). For the application of the simplified procedure, five different seismic inputs were considered, adding progressively one sequence to the next. These inputs were used to derive both the specific elastic response spectra and the corresponding capacity demand spectra for the application of the procedure as indicated above.

Figure $11 \mathrm{~b}$ shows the comparison between the time history of the wall displacements measured in the experiment and the prediction of the simplified method, together with the prediction obtained by Callisto (2019) using the non-linear SDOF model. The agreement obtained with the method proposed in this paper can be deemed quite satisfactory, taking into account the extreme simplifications included in the proposed procedure.

Subsequently, it was also checked that the proposed procedure is effective in the simulation of the response of geotechnical systems that do not reach their capacity during a seismic event. This second validation took as a reference the results of full dynamic numerical analyses reported by Callisto (2019). Figure 12a, b illustrates the case of a bridge abutment founded on piles, that was analysed using a combination of three-dimensional and plane strain finite difference models, subjected to the Tolmezzo seismic record. Figure 12c shows the time-history of the displacement computed with a dynamic finite difference (FLAC) analysis and with the non-linear SDOF model, while Fig. 12d shows the results of the simplified method (note that the AD plane has been rotated to make it consistent with the displacement time history). The elastic response spectrum in Fig. 12d was evaluated at the mid-eight of the abutment from a free-field ground response analysis. In this case, it is evident that $R_{\mathrm{T}}$ is larger than one and therefore the permanent displacement coincides with $u_{\mathrm{I}}$. It can be seen that the permanent displacement predicted by the simplified method is nearly coincident with that calculated with the full dynamic analysis, and also the maximum instantaneous displacement obtained in the AD plane $\left(s_{\text {int }} \times H\right)$ is quite close to that obtained with the more complex numerical computation.

A final validation employed the entire set of ground motion records included in the SIMBAD database, taking as a reference the results of the time-domain integration of the non-linear SDOF model presented in Sect. 2.2, which is efficient enough to be applied to 
(a)

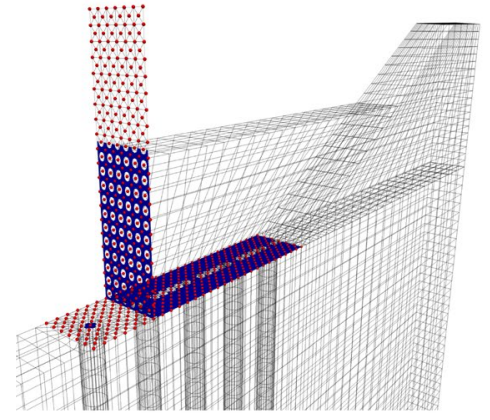

(c)

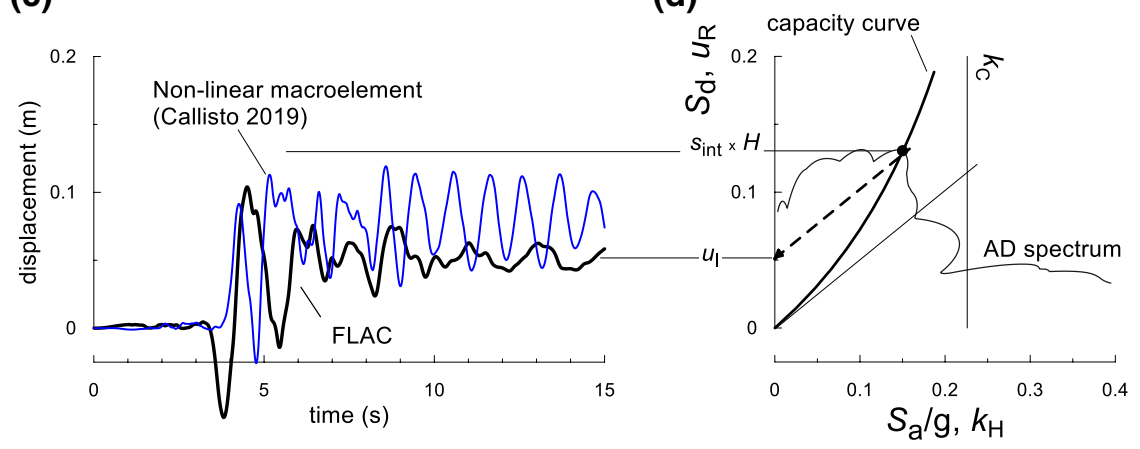

Fig. 12 Finite-difference numerical model of a piled abutment: a three-dimensional model; $\mathbf{b}$ equivalent plane-strain model. Results of the computations: c plane-strain numerical model and non-linear SDOF; d simplified procedure

a large number of cases. In this validation, three abstract geotechnical systems were considered, having the same height $(H=4 \mathrm{~m})$ but three different capacity curves as depicted in Fig. 13. The values of the parameters for these three capacity curves are reported in Table 2.

In a first stage these capacity curves were used to evaluate the permanent displacement through a time-domain integration of the non-linear SDOF (Eq. 3), applying each ground motion with a positive and negative sign and considering the largest of the two computed displacements, for a total of $3 \times 467=1401$ displacement values. Subsequently the same three capacity curves and the elastic response spectra of each record were used to implement the simplified procedure described above, obtaining a different set of values for the permanent displacement of the three systems.

The comparison between the predictions of the simplified method and the time-domain calculations are shown in Fig. 14. Overall, the predictions of the simplified method are quite good, considering that the method condenses the effect of a complex time-history into an elastic response spectrum and an average cyclic capacity spectrum. It is remarkable that a good agreement is obtained over a very wide interval of displacements, ranging from $0.01 \%$ to $50 \%$ of the height $H$ of the system. The average error, indicated with the thin lines in the figure, is equal to $54 \%$. However, it is also interesting to observe that the error gets smaller (in the average equal to $40 \%$ ) when the displacements become larger than, say, $1 \%$ of the height of the system, that is, for the cases of more practical significance in design. 
Fig. 13 Capacity curves used for the validation of the simplified procedure

Table 2 Parameters of the capacity curves used for the validation of the simplified model

Fig. 14 Comparison of the results of the simplified method with the results of time-domain calculations for the entire SIMBAD database

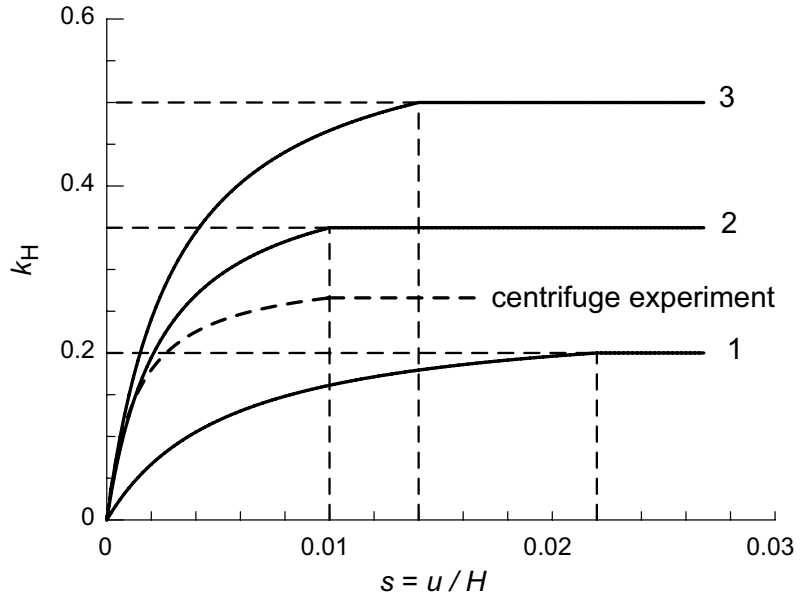

\begin{tabular}{lllllll}
\hline $\begin{array}{l}\text { Capacity } \\
\text { curve }\end{array}$ & $k_{\mathrm{C}}$ & $s_{\mathrm{C}}$ & $\alpha$ & $\beta$ & $D_{0}$ & $T_{0}(\mathrm{~s})$ \\
\hline 1 & 0.20 & 0.022 & 0.80 & 1.0 & 45.5 & 0.6 \\
2 & 0.35 & 0.010 & 0.80 & 1.0 & 175.0 & 0.3 \\
3 & 0.50 & 0.014 & 0.82 & 2.0 & 198.0 & 0.2 \\
\hline
\end{tabular}

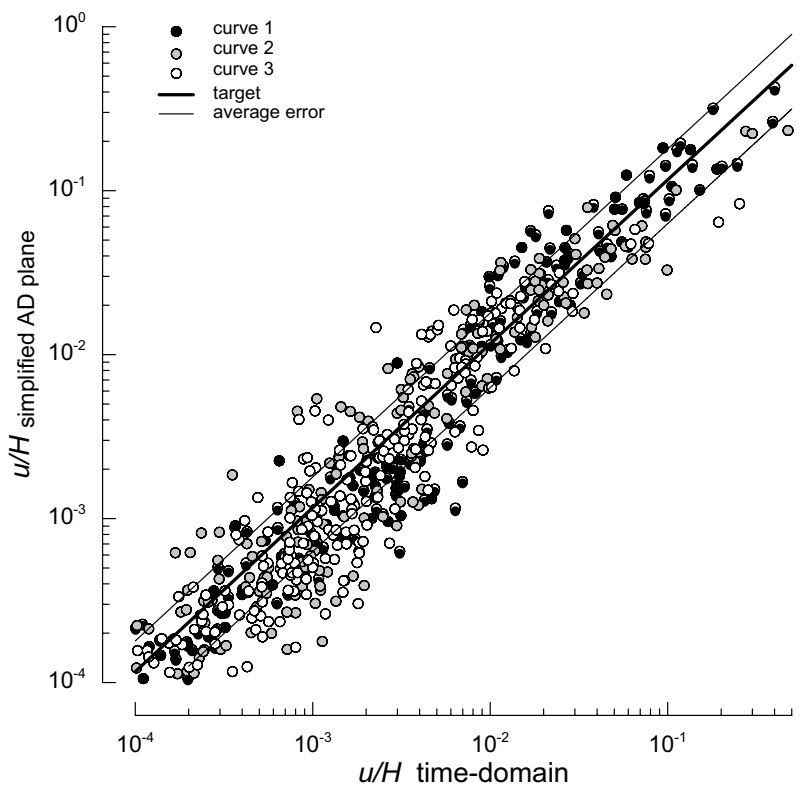


Fig. 15 Average 5\%-damped response spectra of the six group of records used to validate the simplified procedure, and corresponding code spectra

Table 3 Groups of records used to validate the simplified procedure using code spectra

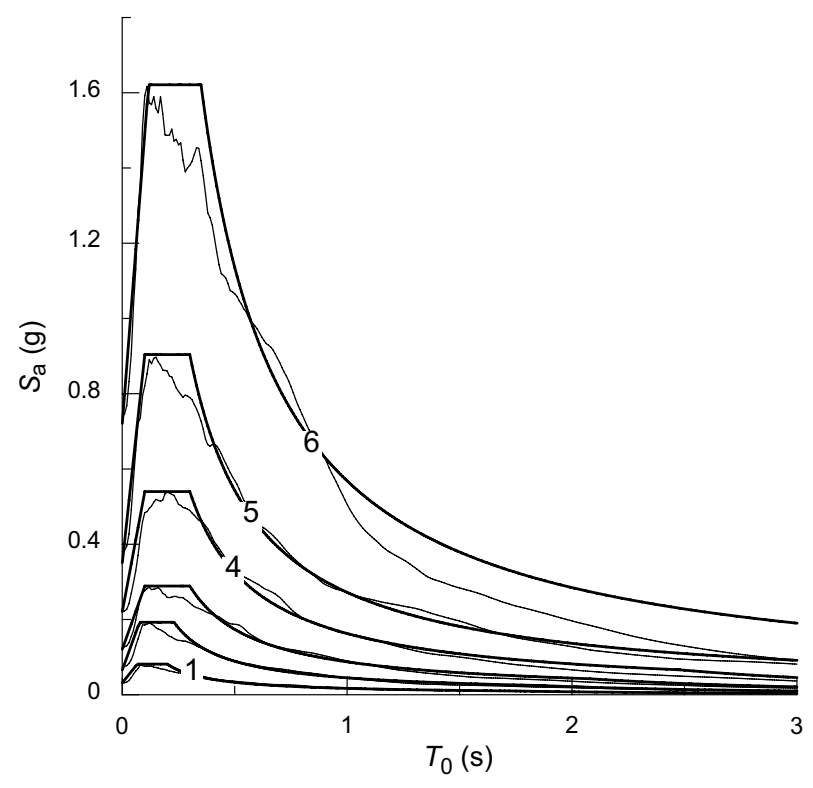

\begin{tabular}{llc}
\hline Group & PGA $(\mathrm{g})$ & No. of records \\
\hline 1 & $<0.05$ & 89 \\
2 & $0.05-0.10$ & 101 \\
3 & $0.10-0.15$ & 79 \\
4 & $0.15-0.3$ & 105 \\
5 & $0.30-0.5$ & 56 \\
6 & $0.5-2.0$ & 37 \\
\hline
\end{tabular}

The figure also shows that the error associated with the three different systems is fairly uniform, indicating that the proposed method has a similar reliability for systems with quite different properties (see Table 2).

\section{Use of code spectra}

Once the method has been validated looking at the response for actual dynamic records, it is necessary to test its validity for design purposes, using code spectra to express the seismic demand, also in view of the fact that the normalized shape of code spectra entails some loss of information due to their regular shape (Freeman 2007). To this end, the SIMBAD records were collected into six groups based on their maximum acceleration (PGA), and the average elastic response spectrum of each group was described using spectral shapes provided by the Italian Technical Code. Figure 15 shows a comparison of the average response spectra of each group and the corresponding code spectra, with reference to a damping ratio of 5\%, while Table 3 provides the PGA intervals and number of records considered for each group. 
Fig. 16 Validation of the simplified procedure using the code spectra of Fig. 15

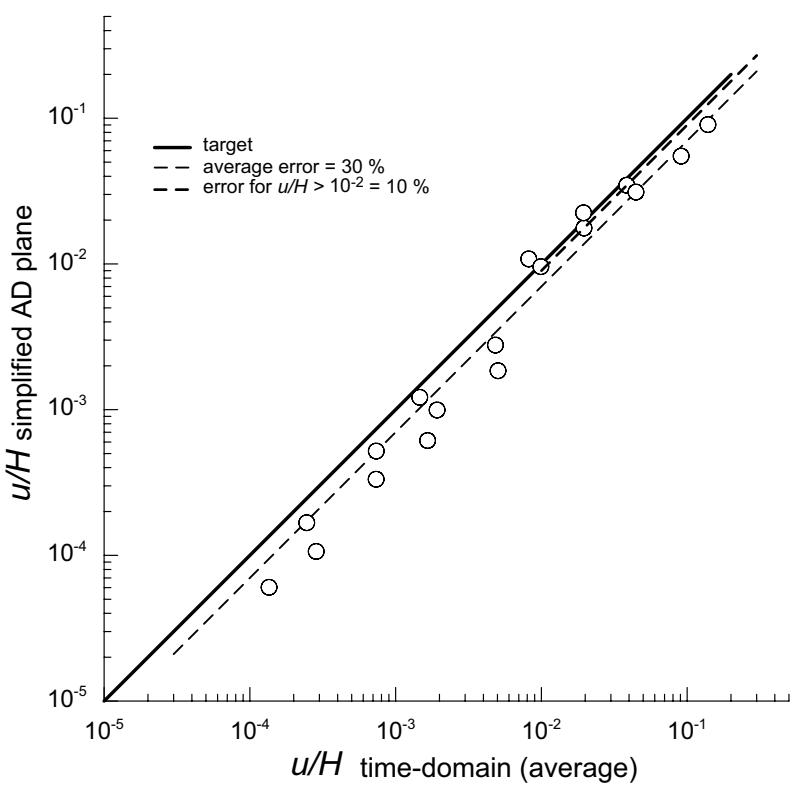

Subsequently, the geotechnical systems characterised by the three capacity curves of Table 2 were analysed using the spectral shapes of Fig. 15 as a seismic demand, conjunctly with the cyclic demand spectra for Fig. 10, obtaining for each system six different displacements. In the iterations in the A-D plane, the code spectra were adjusted for damping ratios different from $5 \%$ multiplying the spectral ordinates by the damping correction factor (CEN 2004):

$$
\eta=\sqrt{\frac{10}{5+\xi}}
$$

In Fig. 16, these displacements are compared to those obtained from the time-domain integration of Eq. (3), averaging the results obtained for each group of records. It can be seen that the average error is of the order of $30 \%$. Specifically, for displacements larger than $1 \%$ of $H$ the agreement is quite good, with an average error of about $10 \%$, while the simplified method underestimates the displacements by about $45 \%$ for smaller displacements. In practice this under-estimate has little relevance because it involves very small displacements. A closer inspection of the results revealed that the error in this displacement range derives from an under-estimate of the first-activation displacement $u_{\mathrm{I}}$ of Fig. $6 \mathrm{~b}$ which is quite sensitive to the approximate adjustment of the spectral ordinated provided by Eq. (13).

\section{Conclusions}

When subjected to seismic actions, the geotechnical systems considered in this paper exhibit a strongly non-linear behaviour related to the progressive activation of their capacity, that in turn may produce a significant accumulation of displacements: the seismic performance for this category of constructions is in fact expressed by a measure of 
its final, permanent deformation. In principle the permanent displacements should be evaluated using a time-domain analysis of a suitable numerical model of the system, including the structural members and the surrounding soils. It is also possible to employ simpler time-domain analysis, either inspired by the Newmark (1965) method, that assume a rigid-perfectly plastic response of the system, or incorporating a simplified description of the dynamic response of the system, like the one developed by Callisto (2019). However, any type of time-domain analysis requires a representation of the seismic action through time histories, and for simple structures this represents a significant burden to the designer. The objective of this paper was to propose a simplified method that, retaining the essential features of the seismic behaviour of the systems under consideration, could be at the same time routinary employable in design.

Because of the non-linearity of their response, it was tempting to extend to the systems at hand the tools that are commonly used to perform non-linear static analysis of structures. In fact, it was shown that the seismic capacity of the geotechnical systems herein considered can be represented quite effectively through appropriate capacity curves. However, the one-directional accumulation of displacements called for an extension of the usual AD-plane analysis, that required the development of specific, threshold-dependent cyclic demand spectra. For the purpose of this work these cyclic demand spectra were related to the specific seismicity of the Italian territory. Once the preliminary work of deriving region-specific cyclic capacity spectra was completed, then the static non-linear analysis of the geotechnical systems became immediate, requiring a negligible computational effort. In the previous sections it was demonstrated that the proposed procedure is robust and reliable, as it was seen to reproduce quite accurately the results of time-domain calculations over a very wide range of displacements, and was also capable to predict the results of both a complex centrifuge test and a dynamic analysis of a full numerical model.

As a final remark, it is worth mentioning that the superposition of the capacity curve onto the elastic response spectrum in the AD plane can also be useful to evaluate the internal forces in the structural members interacting with the soil: a number of comparisons with results of numerical analyses, not shown here for the sake of brevity, showed that the maximum internal forces in retaining structures are clearly related to the spectral acceleration corresponding to the intersection of the capacity curve with the AD elastic spectrum.

Funding Open access funding provided by Università degli Studi di Roma La Sapienza within the CRUICARE Agreement.

Open Access This article is licensed under a Creative Commons Attribution 4.0 International License, which permits use, sharing, adaptation, distribution and reproduction in any medium or format, as long as you give appropriate credit to the original author(s) and the source, provide a link to the Creative Commons licence, and indicate if changes were made. The images or other third party material in this article are included in the article's Creative Commons licence, unless indicated otherwise in a credit line to the material. If material is not included in the article's Creative Commons licence and your intended use is not permitted by statutory regulation or exceeds the permitted use, you will need to obtain permission directly from the copyright holder. To view a copy of this licence, visit http://creativecommons.org/licenses/by/4.0/.

\section{References}

Ambraseys NN, Menu JM (1988) Earthquake-induced ground displacements. Earthq Eng Struct Dyn 16:985-1006. https://doi.org/10.1002/eqe.4290160704 
ATC (1996) ATC-40 Seismic evaluation and retrofit of concrete buildings. Applied Technology Council, Redwood city

Baziar MH, Rezaeipour H, Jafarian Y (2012) Decoupled solution for seismic permanent displacement of earth slopes using deformation-dependent yield acceleration. J Earthq Eng 16:917-936. https://doi. org/10.1080/13632469.2012.689119

Callisto L (2014) Capacity design of embedded retaining structures. Geotechnique 64:204-214. https://doi. org/10.1680/geot.13.P.091

Callisto L (2019) On the seismic design of displacing earth retaining systems. In: Earthquake geotechnical engineering for protection and development of environment and constructions. In: Proceedings of the 7 th international conference on earthquake geotechnical engineering. Associazione Geotecnica Italiana, Rome, pp 239-255

Callisto L, Rampello S (2013) Capacity design of retaining structures and bridge abutments with deep foundations. J Geotech Geoenviron Eng 139:1086-1095. https://doi.org/10.1061/(ASCE)GT.19435606.0000825

Callisto L, Soccodato FM (2010) Seismic design of flexible cantilevered retaining walls. J Geotech Geoenviron Eng 136:344-354. https://doi.org/10.1061/(ASCE)GT.1943-5606.0000216

CEN (2004) EN1998:1 Eurocode 8: Design of structures for earthquake resistance-part 1: general rules, seismic actions and rules for buildings. Bruxelles: European Committe for standardization. The European Union Per Regulation 305/2011, Directive 98/34/EC, Directive 2004/18/EC

Chopra AK (1966) Earthquake effects on dams. Ph.D. Thesis. University of California, Berkeley, USA

Circolare n.7 21 Gennaio (2019) Istruzioni per l'applicazione delle 'Norme Tecniche per le Costruzioni' di cui al D.M. 17/01/2018. [Instructions for the applications of italian building code D.M. 17/01/2018] Italian Ministry of Infrastructure and Transportation, Rome, Italy. In Italian

Coffin LFJ (1954) A study of the effect of cyclic thermal stresses in ductile metals. Trans ASME 76:931-950

Conti R, Viggiani GMB (2012) Evaluation of soil dynamic properties in centrifuge tests. J Geotech Geoenviron Eng 138:850-859. https://doi.org/10.1061/(ASCE)GT.1943-5606.0000659

Conti R, Madabushi GSP, Viggiani GMB (2012) On the behaviour of flexible retaining walls under seismic actions. Geotechnique 62:1081-1094. https://doi.org/10.1680/geot.11.P.029

Fajfar P (2000) A nonlinear analysis method for performance-based seismic design. Earthq Spectra 16:573592. https://doi.org/10.1193/1.1586128

FEMA (2005) FEMA 440 improvement of nonlinear static seismic analysis procedures. Federal Emergency Management Agency, Redwood City

Franklin AG, Chang FK (1977) Permanent displacements of earth embankments by Newmark sliding block analysis. Earthquake Resistance of earth and rock-fill dams Report n.5. U.S. Army Engineer Waterways Experiment Station, Vicksburg, Mississipi, USA

Freeman SA (1998) Development and use of capacity spectrum method. In: Proceedings of the 6th US National Conference on Earhquake Engineering. Seattle, Washington, USA

Freeman SA (2004) Review of the development of the capacity spectrum method. ISET J Earthq Technol 41:1-13

Freeman SA (2007) Response spectra as a useful design and analysis tool for practicing structural engineers. ISET J Earthq Technol 44:25-37

Freeman SA, Nicoletti JP, Tyrell JV (1975) Evaluations of existing buildings for seismic risk-a case study of Puget sound naval shipyard, Bremerton, Washington. In: Proceedings of the 1st US National conference on earthquake engineering, Berkeley, USA

Malhotra PK (2002) Cyclic-demand spectrum. Earthq Eng Struct Dyn 31:1441-1457. https://doi. org/10.1002/eqe.171

Manson SS (1954) Behavior of materials under conditions of thermal stress. NACA-Technical Note 2933

Newmark NM (1965) Effects of earthquakes on dams and embankments. Geotechnique 15:139-160. https:// doi.org/10.1680/geot.1965.15.2.139

Rathje EM, Bray JD (1999) An examination of simplified earthquake-induced displacement procedures for earth structures. Can Geotech J 36:72-87. https://doi.org/10.1139/t98-076

Rathje EM, Abrahamson NA, Bray JD (1998) Simplified frequency content estimates of earthqauke ground motions. J Geotech Geoenviron Eng 124:150-159

Richards RJ, Elms DG (1979) Seismic behaviour of gravity retaining walls. J Geotech Eng Div 105:449-464

Richards RJ, Elms DG (1992) Seismic passive resistance of tied-back walls. J Geotech Eng 118:996-1011

Richards R, Elms DG, Budhu M (1993) Seismic bearing capacity and settlements of foundations. J Geotech Eng 119:662-674. https://doi.org/10.1061/(ASCE)0733-9410(1993)119:4(662)

Seed BH, Martin GR (1966) The seismic coefficient in earth dam design. J Soil Mech Found Div 92:25-58

Smerzini C, Galasso C, Iervolino I, Paolucci R (2014) Ground motion record selection based on broadband spectral compatibility. Earthq Spectra 30:1427-1448. https://doi.org/10.1193/052312EQS197M 
Veletsos A, Newmark NM (1960) Effect of inelastic behavior on the response of simple systems to earthquake motions. In: Proceedings of the 2nd world conference on earthquake engineering. Tokyo, Japan, pp. 895-912

Whitman RV (1990) Seismic design and behavior of gravity retaining walls. In: Proceedings of ASCE specialty conference on design and performance of earth retaining strucutres, pp 817-834

Wong CP (1982) Seismic analysis and an improved seismic design procedure for gravity retaining walls. M.Sc. Thesis. Massachussets Institute of Technology M.I.T

Publisher's Note Springer Nature remains neutral with regard to jurisdictional claims in published maps and institutional affiliations. 